\title{
Maternal asthma is associated with reduced lung function in male infants in a combined analysis of the BLT and BILD cohorts
}

\author{
Patricia de Gouveia Belinelo, ${ }^{1,2}$ Adam M Collison (D) , 1,2 Vanessa E Murphy (D) , 1,2 \\ Paul D Robinson (D) , 3,4 Kathryn Jesson, ${ }^{1}$ Kate Hardaker, ${ }^{3}$ \\ Ediane de Queiroz Andrade, ${ }^{1,2}$ Christopher Oldmeadow, ${ }^{2}$ \\ Gabriela Martins Costa Gomes, ${ }^{1,2}$ Peter D Sly (D) , Jakob Usemann (D) , 6,7 \\ Rhea Appenzeller, 6,7 Olga Gorlanova, 6,7 Oliver Fuchs, ${ }^{6,7}$ Philipp Latzin, 6,7 \\ Peter G Gibson, $2,8,9$ Urs Frey, ${ }^{6,7}$ Joerg Mattes ${ }^{1,2,10}$
}

- Additional material is published online only. To view please visit the journal online (http://dx.doi.org/10.1136/ thoraxinl-2020-215526).

For numbered affiliations see end of article.

\section{Correspondence to}

Professor Joerg Mattes, Priority Research Centre GrowUpWell, The University of Newcastle, Callaghan, New South Wales, Australia;

joerg.mattes@newcastle.edu. au and Professor Urs Frey, Department of Pulmonology, University Children's Hospital (UKBB), Basel, Switzerland: urs.frey@ukbb.ch

Received 11 June 2020 Revised 26 January 2021 Accepted 12 February 2021 Published Online First 25 February 2021

\section{Linked}

- http://dx.doi.org/10.1136/ thoraxjnl-2021-217095

\section{Check for updates}

(C) Author(s) (or their employer(s)) 2021. No commercial re-use. See rights and permissions. Published by BMJ.

To cite: de Gouveia Belinelo P, Collison AM, Murphy VE, et al. Thorax 2021;76:996-1001.

\section{ABSTRACT}

Rationale Asthma in pregnancy is associated with respiratory diseases in the offspring.

Objective To investigate if maternal asthma is associated with lung function in early life.

Methods Data on lung function measured at 5-6 weeks of age were combined from two large birth cohorts: the Bern Infant Lung Development (BILD) and the Australian Breathing for Life Trial (BLT) birth cohorts conducted at three study sites (Bern, Switzerland; Newcastle and Sydney, Australia). The main outcome variable was time to reach peak tidal expiratory flow as a percentage of total expiratory time(tPTEF:tE\%). Bayesian linear hierarchical regression analyses controlling for study site as random effect were performed to estimate the effect of maternal asthma on the main outcome, adjusting for sex, birth order, breast feeding, weight gain and gestational age. In separate adjusted Bayesian models an interaction between maternal asthma and sex was investigated by including an interaction term.

Measurements and main results All $406 \mathrm{BLT}$ infants were born to mothers with asthma in pregnancy, while 193 of the 213 (91\%) BILD infants were born to mothers without asthma. A significant interaction between maternal asthma and male sex was negatively associated with tPTEF:tE\% (intercept 37.5; estimate: -3.5; 95\% credible interval -6.8 to -0.1$)$. Comparing the model posterior probabilities provided decisive evidence in favour of an interaction between maternal asthma and male sex (Bayes factor 33.5).

Conclusions Maternal asthma is associated with lower lung function in male babies, which may have lifelong implications on their lung function trajectories and future risk of wheezing and asthma.

\section{INTRODUCTION}

Maternal asthma is the most common medical condition in pregnancy, and uncontrolled asthma during pregnancy increases the risk of placental complications, ${ }^{1}$ low birth weight, prematurity ${ }^{2}{ }^{3}$ and asthma development in the offspring. ${ }^{4} \mathrm{~A}$ better understanding of its effect on the offspring's lung function in early life may provide a physiological

\section{Key messages}

What is the key question?

- Do babies born to mothers with asthma have reduced lung function in early life?

What is the bottom line?

- Male babies born to mothers with asthma have a significantly lower lung function, which may affect their lung function trajectory and risk of developing respiratory disease.

Why read on?

- This analysis included 619 babies from three study sites, $69 \%$ of them born to a mother with asthma, and identified an interaction between maternal asthma and male sex as an early determinant of lung function.

mechanism for the well-established associations between maternal asthma and adverse respiratory health outcomes in their offspring. Existing literature on the impact of maternal asthma on infant lung function during the first 6 weeks of life is limited to unselected birth cohorts which included babies born to mothers/families with an asthma incidence of 9\%-14\%. ${ }^{56}$ In the Western Australian Pregnancy Cohort Study, lung function was measured by respiratory inductance plethysmography within the first 48 hours of life. A family history of asthma was associated with significantly lower values of time to reach peak tidal expiratory flow as a ratio of total expiratory time (tPTEF:tE) ${ }^{6}$ an integrated output of the entire respiratory system, including airflow limitation and control of breathing. ${ }^{7}$

Lung function in early life predicts future risk of respiratory diseases and tracks throughout life..$^{8-10}$ However, an association between maternal asthma and lung function parameters at 5-6weeks of age has not been investigated. We combined tidal breathing flow-volume loop (TBFVL) test data from an Australian birth cohort of 6-week-old infants, all born to mothers with asthma in pregnancy (Breathing for Life Trial (BLT)), ${ }^{11}$ with data 
from the Bern Infant Lung Development (BILD) cohort, an unselected birth cohort of 5 -week-old infants. ${ }^{5}$ Our primary aim was to investigate the association between maternal asthma and $\mathrm{tPTEF} / \mathrm{tE} \%$.

\section{METHODS}

\section{Study participants}

In the BLT study, babies were recruited from mothers with mild to moderate asthma in pregnancy who participated in a randomised asthma management intervention during pregnancy. ${ }^{11}$ Asthma in pregnancy was defined as self-reported, doctor-diagnosed asthma and current asthma symptoms or inhaled asthma medication use. Maternal asthma was of mild to moderate severity according to the Global Initiative for Asthma (GINA) guidelines. BLT women who had used oral corticosteroids for more than 14 days in the 3 months prior to study enrolment were excluded. During pregnancy 51 BLT mothers had asthma exacerbations, of whom 28 presented to their general practitioner and 1 woman presented to the hospital for her asthma exacerbation. Of the 51 mothers 18 had oral corticosteroids (OCS) prescribed for severe exacerbations.

If consent was given by parent(s)/guardian(s), infants were prospectively followed up in Newcastle, Sydney and Brisbane, Australia, with measurements including lung function at 6 weeks corrected for gestational age (Newcastle and Sydney only), clinical assessment (all sites) and parent-reported questionnaire at 6 and 12 months of age. Infants at 6 weeks of age were seen between May 2014 and December 2019. The inclusion criterion for infant lung function testing was no apparent major birth defects or perinatal disease that would preclude performing unsedated infant lung function. Infants born preterm were included. Written informed parental consent was obtained at enrolment.

Data from the BILD study included babies recruited between 1999 and 2010 in Bern, Switzerland. ${ }^{5}$ A history of maternal asthma was defined as self-reported, doctor-diagnosed asthma. In the BILD cohort maternal asthma was also of mild to moderate severity. Nine mothers used inhaled corticosteroids during pregnancy. In contrast to BLT, only babies born at term delivery ( $\geq 37$ weeks) were included. In accordance with BLT, only babies who did not have apparent major birth defects or perinatal disease that would preclude infant lung function testing at 5 weeks of age were included. Written informed parental consent was obtained at enrolment.

Known and potential predictors of lung function (demographic data, sociodemographic status) were assessed by interviews and by using standardised questionnaires in both the BLT and BILD studies. For further information on similarities and differences between the studies, see also online supplemental table E1.

\section{Lung function}

Both cohorts had lung function measured using equipment from identical suppliers in unsedated infants during behaviourally defined quiet natural sleep. They were performed with the infants lying supine, using an infant mask (sizes $0,0 / 1$ and 1; Homedica, Huenenberg, Switzerland), according to the European Respiratory Society/American Thoracic Society (ERS/ATS) standards of infant lung function testing, ${ }^{12} 13$ and the mask size dead space was corrected during analysis. Flow was measured using an ultrasonic flow metre (Spiroson; Eco Medics, Duernten, Switzerland). Data were included if no apparent volume drift, defined as a change of $<3 \mathrm{~mL} / \mathrm{s}$ over at least 30 breaths, was present. Both cohorts performed infant lung function following similar protocols based on recommended quality criteria, ${ }^{13}$ differing only with regard to the duration of the tests. In the BILD study, TBFVL was performed for 10 min with the purpose of obtaining 100 good-quality breaths, while in the BLT study TBFVL was performed for $90 \mathrm{~s}$ with the aim of obtaining at least 30 good-quality breaths. ${ }^{14}$

Raw data from both cohorts were reanalysed using a protocol designed to preclude between-site differences. The relevant staff member (PDGB) was extensively trained at all study sites (Newcastle, Sydney, Bern) by the investigators before conducting analyses. PDGB performed the site analyses at different dates, and data were not masked with regard to site and maternal asthma. Mean TBFVL measures for the two cohorts combined were calculated using an identical software version (Wbreath V.3.28.0; Ndd Medizintechnik, Zurich, Switzerland) for both studies. Records were accepted if they included $\geq 30$ regular breaths of tidal breathing during quiet sleep. In addition, sighs (defined as a marked increase (at least double) in tidal volume with no other artefacts present) were excluded. Mean tidal breathing parameters of flow, volume and flow-volume loop were then calculated according to the ERS/ATS standards. ${ }^{15}$ The outcome parameter reported in the main manuscript is tPTEF:tE\%.

\section{Statistical analysis}

Descriptive statistics and regression analyses were performed using Stata SE V.15 for Windows and R V.4.0 (The R Core Team, Vienna, Austria). ${ }^{16}$ Differences between infant characteristics for those born to mothers with and without asthma during pregnancy were assessed using $\chi^{2}$ tests (for categorical variables) and independent sample t-tests (for continuous variables). A p value $<0.05$ was considered significant.

The effect of maternal asthma on infant lung function (tPTEF:tE\%), controlling for study sites (Bern, Newcastle, Sydney), was assessed using a Bayesian linear hierarchical regression model. The Bayesian hierarchical model was chosen due to the low number of sites and the ability to choose a flexible prior distribution for the random effect prior distribution. ${ }^{17}$ The model included tPTEF:tE\% as the outcome variable and adjusted for confounding variables as fixed effects. The confounders included in the final models (sex, gestational age and weight gain from birth to test date, age at test date in days, birth order, exclusive breast feeding) were identified using backward selection out of a larger list of variables (sex, gestational age, weight gain from birth to test date, weight and length at test date, age at test date in days, birth order, delivery mode, maternal tobacco exposure during pregnancy and exclusive breast feeding until the day of test). Study site was modelled as a random effect assuming normal distribution. Between-site SD was modelled with a halfnormal prior, and vague prior distributions for all the fixed effect parameters (normal distributions centred on zero with a wide variance of 100). We also assessed for the effect of interaction between maternal asthma and infant sex, fitting a model with and without the interaction term and calculating the Bayes factor, representing the ratio of the two posterior model probabilities. A Bayes factor greater than 20 can be interpreted as decisive evidence in favour of the first model where the interaction term was included. ${ }^{18}$ All regression models had converged with 25000 iterations. Samples from the posterior distributions of the regression models were obtained using the No-U-Turn Sampler and the R package BRMS ${ }^{19}$ with four chains to enable calculation of the R-hat convergence statistics. Further plots were constructed to explore convergence, including histograms and 
Table 1 Baseline characteristics and TBFVL parameters in all BLT and BILD study infants, stratified for term pregnancy and asthma in pregnancy

\begin{tabular}{|c|c|c|c|c|c|c|}
\hline & $\begin{array}{l}\text { Maternal asthma, } \\
\text { term infants } \\
\text { (BLT cohort, } n=373 \text { )* }\end{array}$ & $\begin{array}{l}\text { No maternal asthma, } \\
\text { term infants } \\
\text { (BILD cohort, } n=193 \text { ) }\end{array}$ & & $\begin{array}{l}\text { Maternal asthma, } \\
\text { preterm infants } \\
\text { (BLT cohort, } n=33 \text { )* }\end{array}$ & $\begin{array}{l}\text { Maternal asthma, term } \\
\text { infants } \\
\text { (BILD cohort, } n=20 \text { ) }\end{array}$ & \\
\hline & Mean (SD) & Mean (SD) & $P$ valuet & Mean (SD) & Mean (SD) & $P$ value $\neq$ \\
\hline tPTEF:tE\%§ & $32.00(9.8)$ & $35.00(10.5)$ & 0.0022 & $28.55(8.4)$ & $34.45(8.8)$ & 0.1603 \\
\hline Birth order & $1.7(1.0)$ & $1.7(0.8)$ & 0.2299 & $1.6(0.9)$ & $1.5(0.8)$ & 0.5826 \\
\hline Age (days) & $48.1(10.8)$ & $35.8(5.2)$ & $<0.0001$ & $65.2(22.6)$ & $36.9(5.6)$ & $<0.0001$ \\
\hline Weight gain (kg per day) & $0.031(0.012)$ & $0.028(0.011)$ & 0.0763 & $0.030(0.008)$ & $0.025(0.009)$ & 0.0544 \\
\hline \multirow[t]{2}{*}{ Maternal age (years) } & $30.0(5.2)$ & $32.5(4.2)$ & $<0.0001$ & $30.2(4.4)$ & $32.4(2.9)$ & 0.0200 \\
\hline & n (\%) & n (\%) & & n (\%) & $\mathrm{n}(\%)$ & \\
\hline Male sex & $190(51)$ & $101(52)$ & 0.7904 & $19(57)$ & $13(65)$ & 0.2561 \\
\hline Exclusive breast feeding & $224(59)$ & 181 (94) & $<0.0001$ & $17(48)$ & $19(95)$ & 0.0014 \\
\hline Tobacco exposure in pregnancy & $37(10)$ & $17(9)$ & 0.7634 & $4(12)$ & 0 & \\
\hline Caesarean section & $107(29)$ & $30(15)$ & 0.0004 & $26(81)$ & $9(45)$ & 0.1347 \\
\hline
\end{tabular}

BLT infants were all born to mothers with asthma, stratified for term and preterm. BILD infants were term born babies, stratified for no maternal asthma and maternal asthma.

* Maternal asthma, term infants (BLT) $n=373$; and maternal asthma, preterm infants (BLT) $n=33.1$ BLT infant did not have perinatal outcomes available.

tComparing maternal asthma, term infants from BLT cohort versus no maternal asthma and term infants from BILD cohort. P values $<0.05$ in bold.

‡Comparing maternal asthma, term infants from BLT cohort versus maternal asthma and term infants from BILD cohort. P values $<0.05$ in bold.

§tPTEF:tE\%=percentage time to peak tidal expiratory flow divided by total expiratory time.

BILD, Bern Infant Lung Development; BLT, Breathing for Life Trial; TBFVL, tidal breathing flow-volume loop.

scatter plots of the posterior samples of all model parameters. Mean posterior estimates are presented together with $95 \%$ credible intervals, R-hat statistics and effective sample sizes.

\section{RESULTS}

Between May 2014 and December 2019, 1035 eligible infants were born to mothers participating in BLT at the Newcastle and Sydney study sites where lung functions measurements were performed. Of those infants, consent was obtained for 662 offspring $(64 \%)$ to participate in the birth cohort follow-up (online supplemental figure E1). Five hundred and ninety infants $(89 \%)$ had lung function measurements taken at 6 weeks of age, and technically acceptable data were obtained for 406 (69\%) babies. The BILD cohort recruited 365 infants antenatally from 1999 to 2010. Of these, 342 (94\%) babies had infant lung function measured at 5 weeks of age, and 213 (62\%) technically acceptable measurements were obtained. This provided a combined number of 619 infant lung function tests for analysis in this study. The proportions of infants contributing data across the two cohorts are summarised in online supplemental figures E1 and E2 for BLT and BILD, respectively.

Eight per cent of the BLT babies, all born to mothers with asthma in pregnancy, were premature. Nine per cent of the BILD babies, all delivered at term as per inclusion criteria of this study, were born to mothers with asthma. A comparative analysis between term infants born to mothers with asthma and mothers without asthma (table 1, left two columns) and between infants delivered term and preterm (table 1, right two columns) revealed multiple differences (further variables shown in online supplemental table E2). This included differences in the main outcome variable tPTEF: $\mathrm{E} \%$, with lower values observed in babies born at term to mothers with asthma as compared with those born to mothers without asthma (table 1). tPTEF:tE\% also trended towards lower values in male babies as compared with female babies (mean/SD 32.2/10.0 for male babies $(n=323)$ vs 33.5/9.7 for female babies $(n=296), p=0.05)$. Furthermore, significant differences in a number of variables associated with $\mathrm{TPTEF}: \mathrm{tE} \%$ were observed between the three study sites (online supplemental table E3). Therefore, a multivariable Bayesian linear hierarchical regression model was used to correct for study site as random effect in order to estimate the effect of maternal asthma, the infant's sex and its interaction on tPTEF:tE\%. First, a model without including an interaction term between maternal asthma and the infant's sex ("maternal asthma:male sex") was performed, followed by including the interaction term (table 2). When comparing the model posterior probabilities of the two models with the Bayes factor, the model with the interaction term was 33.5 times more probable than the model without the interaction, indicating decisive evidence in favour of an interaction. The $95 \%$ credible interval of the interaction term excluded zero (table 2), thereby confirming that the interaction between maternal asthma and male infant's sex predicted a lower tPTEF:tE\% even when correcting for study site and adjusting for confounders. Age at test, weight gain and birth order were also associated with tPTEF:tE\% (table 2). The results were comparable when only including babies born at term in the Bayesian analysis (table 3), again indicating decisive evidence in favour of an interaction between maternal asthma and the infant's sex (Bayes factor 37.1).

\section{DISCUSSION}

This study combined data from two large prospective multicentre birth cohorts to investigate the effect of a history of maternal asthma on lung function in early life assessed using TBFVL analysis. A statistical interaction between maternal asthma and infant's sex was found, suggesting that a history of maternal asthma was associated with a lower $\mathrm{tPTEF}: \mathrm{tE} \%$ in male infants only. Furthermore, age at test, weight gain and birth order were associated with $\mathrm{tPTEF} / \mathrm{tE} \%$.

As all offspring in Newcastle and Sydney were born to mothers with asthma, the analysis was complicated by collinearity between the study site and the maternal asthma variable. To resolve this, we used Bayesian linear hierarchical regression models that provided decisive evidence for an interaction between maternal asthma history and infant sex when study sites were controlled for as random effects.

It is possible that the negative effect of maternal asthma history on $\mathrm{tPTEF}: \mathrm{tE} \%$ diminishes in female babies in the first weeks of 
Table 2 Predictors of tPTEF:tE\% in a Bayesian linear hierarchical regression model in a combined data set of all BLT and BILD study infants (born term and preterm)

\begin{tabular}{|c|c|c|c|c|c|c|}
\hline Total infants, $\mathrm{N}=619$ & Estimate & Estimate error & $\begin{array}{l}95 \% \text { credible } \\
\text { interval }\end{array}$ & R-hat & Bulk ESS & Tail ESS \\
\hline Intercept & 37.49 & 2.47 & 32.39 to 42.21 & 1.00 & 10871 & 7632 \\
\hline Maternal asthma & 0.57 & 1.89 & -2.94 to 4.66 & 1.00 & 8236 & 3675 \\
\hline Male sex & 0.85 & 1.42 & -1.94 to 3.62 & 1.00 & 16964 & 23535 \\
\hline Maternal asthma:male sex & -3.48 & 1.71 & -6.81 to -0.11 & 1.00 & 19033 & 24182 \\
\hline fSecond-born* & -1.95 & 0.94 & -3.79 to -0.09 & 1.00 & 15363 & 9755 \\
\hline Third-born or later* & -1.90 & 1.12 & -4.08 to 0.31 & 1.00 & 17101 & 17230 \\
\hline Age (days) & -0.19 & 0.05 & -0.28 to -0.11 & 1.00 & 20661 & 23030 \\
\hline Exclusive breast feeding & 1.42 & 0.95 & -0.42 to 3.27 & 1.00 & 16140 & 15582 \\
\hline Weight gain $(\mathrm{kg})$ & 3.17 & 0.94 & 1.34 to 5.04 & 1.00 & 21785 & 19535 \\
\hline
\end{tabular}

Bayes factor=33.54.

*First-born is reference.

BILD, Bern Infant Lung Development; BLT, Breathing for Life Trial; ESS, effective sample size; tPTEF:tE\%, time to reach peak tidal expiratory flow as a percentage of total expiratory time.

life but lags behind in male infants. Currently ongoing follow-up studies of our cohorts will be able to test this hypothesis. The trend towards lower tPTEF:tE\% in male infants reported in this study $(p=0.05)$ has been described earlier. ${ }^{20}$ The concept of sexual dimorphism with differences in lung development and growth trajectories between male and female babies is supported by ample epidemiological and experimental data, including risk differences for neonatal lung disease, ${ }^{21}$ and effects of androgens on surfactant production, ${ }^{22}$ airway morphogenesis, ${ }^{23}$ and lung transcriptome data and its regulation. ${ }^{24}$ These data propose that lung development in male fetuses may lag behind that of female fetuses in the late stages of gestation. Our study is in accordance with these concepts, however identifies a greater susceptibility of male babies to the negative effects conferred by maternal asthma on infant lung function outcomes. Sexual dimorphism in lung development and trajectories may be the underpinning mechanism for, or the consequence of, sex differences in susceptibility to adverse effects.

Better lung function in male babies is clinically relevant as it is associated with less wheezing in infancy and asthma in childhood, ${ }^{2526}$ and less severe viral lower respiratory tract infections. ${ }^{27}$ Furthermore, lung function trajectories may be established in early life and persist throughout childhood and beyond. Male sex has been shown to be an independent risk factor for a reduced
tPTEF:tE and reduced respiratory system compliance, with a reduced mean tPTEF:tE after birth being related to history of asthma in the first 10 years of life. ${ }^{28}$ Specifically, Håland et $a l^{28}$ reported a mean tPTEF:tE of 0.32 for the whole infant population $(n=614)$, which is comparable with our results that are expressed as a percentage. Four hundred and ninety participants had no history of asthma at 10 years of age and the mean tPTEF:tE after birth was 0.322 (95\% CI 0.312 to 0.332 ), and 124 children had a history of asthma and the mean tPTEF:tE was 0.298 (95\% CI 0.278 to 0.317 ) after birth. The reduction in mean tPTEF:tE of approximately $10 \%$ in babies who develop asthma at 10 years of age is comparable with the magnitude of reduction in mean tPTEF:tE\% observed in male infants born to mothers with asthma in our study. Bisgaard et $a l^{29}$ found that approximately $40 \%$ of the lung function deficit observed in children with asthma at 7 years of age was already present at birth. Thus, the reduction in tPTEF:tE\% observed in our study may be clinically relevant. Our data suggest that the risk conferred by maternal asthma for the later development of asthma in boys, but not girls, could be modulated by the association between maternal asthma and reduced lung function in early life. Thus, while the association between maternal asthma and persistent wheezing may be independent of the child's sex, ${ }^{30}$ effects of maternal asthma on lung function predisposing to persistent wheezing may only be relevant in boys.

Table 3 Predictors of tPTEF:tE\% in a Bayesian linear hierarchical regression model in a combined data set of BLT and BILD study infants born at term (preterm babies excluded)

\begin{tabular}{|c|c|c|c|c|c|c|}
\hline$n=586$ & Estimate & Estimate error & $95 \%$ credible interval & R-hat & Bulk ESS & Tail ESS \\
\hline Intercept & 37.32 & 2.76 & 31.55 to 42.51 & 1.00 & 5789 & 7576 \\
\hline Maternal asthma & 0.68 & 1.92 & -2.92 to 4.68 & 1.00 & 7449 & 9193 \\
\hline Male sex & 0.79 & 1.42 & -2.00 to 3.55 & 1.00 & 10823 & 13833 \\
\hline Maternal asthma:male sex & -3.64 & 1.73 & -7.06 to -0.24 & 1.00 & 8629 & 13490 \\
\hline Second-born* & -1.80 & 0.98 & -3.70 to 0.14 & 1.00 & 9005 & 2827 \\
\hline Third-born or later* & -1.98 & 1.16 & -4.25 to 0.27 & 1.00 & 12096 & 4590 \\
\hline Age (days) & -0.20 & 0.06 & -0.31 to -0.09 & 1.00 & 14515 & 14057 \\
\hline Exclusive breast feeding & 1.30 & 0.99 & -0.63 to 3.24 & 1.00 & 9189 & 5330 \\
\hline Weight gain $(\mathrm{kg})$ & 3.64 & 0.98 & 1.70 to 5.56 & 1.00 & 13732 & 21417 \\
\hline
\end{tabular}

Bayes factor $=37.12$.

${ }^{*}$ First-born is reference.

BILD, Bern Infant Lung Development; BLT, Breathing for Life Trial; ESS, effective sample size; tPTEF:tE\%, tidal expiratory flow to expiratory time ratio. 
An unexpected result of this study was that we did not find any association between self-reported tobacco exposure during pregnancy and TBFVL parameters. However, Stick and coworkers ${ }^{6}$ found, based on infants' urinary cotinine levels, that approximately $6 \%$ of infants of self-reported non-smoking mothers were likely to have had substantial exposure to tobacco smoke in utero. Their mean tPTEF:tE was lower when compared with infants with low urinary cotinine. Thus, our data re-emphasise the need to objectively quantify intrauterine tobacco smoke exposure to accurately estimate its detrimental effects on lung function and respiratory health in early life. None of the other variables shown in online supplemental table E2 to be differently distributed between the BLT and BILD cohort were significantly associated with tPTEF:tE\%, including caesarean section which was previously associated with lung function outcomes in some but not all cohorts. ${ }^{3132}$

The main strengths of this analysis included a large sample size with a high proportion of infants born to mothers with asthma in pregnancy. This enabled the stratification of analysis by the infant's sex to identify significant lung function predictors and test for statistical interactions. This analysis used comparable TBFVL methodology across study sites for testing (online supplemental table E1) and a stringent approach towards reanalysis of all lung function raw data. Finally, Bayesian linear hierarchical regression analyses allowed controlling for study site as random effect.

One of the limitations of this study was that the BLT cohort did not include infants born to mothers without asthma. However, as the BILD cohort included infants born to mothers with and without asthma, the effect of maternal asthma could be estimated independently from a study site effect. Another possible limitation of our analysis is that we have not correlated lung function outcomes with respiratory symptoms in the first year of life. This is currently under way in the BLT cohort, but those infants are all born to mothers with asthma. The long-term effect of the interaction between maternal asthma and male infant sex on lung function trajectory and respiratory outcomes will be an important topic for future projects.

Additionally, the proportion of BILD mothers with an asthma history who had asthma symptoms or medication in pregnancy is unknown. As asthma may improve in one-third of pregnant women, ${ }^{33}$ this bias may have increased a type 2 error. Finally, the biological mechanisms that underpin the effect of maternal asthma on infant lung function remain elusive. Recent experimental studies have revealed a role of maternal interleukin-5 (IL-5) release in the early origins of bronchoconstriction. ${ }^{34}$ Fetal eosinophils induced by maternal IL-5 promoted airway sensory innervation and epithelial nerve density, resulting in increased vagal reflex-induced bronchoconstriction in the fetal lungs. This also potentiated airways hyperactivity to allergen exposure. ${ }^{35}$ It is possible that uncontrolled eosinophilic asthma in pregnancy may be associated with an increased risk of developing bronchoconstriction in the offspring that is acquired in utero. $^{36}$

Together, our data identified maternal asthma history as a risk factor for impaired lung function in early life in male infants. Considering that lung function follows trajectories and impaired lung function is associated with increased disease risk, our data suggest that further longitudinal follow-up data should be analysed to determine those risks. Furthermore, fetal sex and maternal asthma history should be considered as potential confounders when analysing infant lung function data. Lastly the mechanisms that underpin the association of fetal sex and maternal asthma with lung function at 6 weeks of age should be explored. This finding may inform future preventative strategies aiming at optimising fetal lung development.

\section{Author affiliations}

${ }^{1}$ Priority Research Centre GrowUpWell, University of Newcastle, Callaghan, New South Wales, Australia

${ }^{2}$ Viruses, Infections, Vaccines \& Asthma Research Program, Hunter Medical Research Institute, New Lambton Heights, New South Wales, Australia

${ }^{3}$ Department of Respiratory Medicine, The Children's Hospital at Westmead, Sydney, New South Wales, Australia

${ }^{4}$ Discipline of Paediatrics and Child Health, University of Sydney, Sydney, New South Wales, Australia

${ }^{5}$ Centre for Children's Health Research, University of Queensland, South Brisbane,

Queensland, Australia

${ }^{6}$ Department of Pulmonology, University Children's Hospital (UKBB), Basel,

Switzerland

${ }^{7}$ Paediatric Respiratory Medicine, Department of Paediatrics, Inselspital, University of Bern, Bern, Switzerland

${ }^{8}$ Priority Research Centre for Healthy Lungs, University of Newcastle, Callaghan, New South Wales, Australia

${ }^{9}$ Department of Respiratory and Sleep Medicine, John Hunter Hospital, New Lambton Heights, New South Wales, Australia

${ }^{10}$ Department of Paediatric Respiratory and Sleep Medicine, John Hunter Children's

Hospital, New Lambton Heights, New South Wales, Australia

Twitter Paul D Robinson @DrPLungResearch

Acknowledgements We wish to thank and acknowledge the entire BILD and BLT teams for their invaluable contribution. We would also like to thank Dr Biarta RhysJones, Dr Ana Pereira de Siqueira and Kelly Steel from the University of Newcastle and John Hunter Children's Hospital. In addition, we would like to acknowledge the contribution of Dr Kathryn Ramsey, Dr Andras Soti and Dr Johanna Kurz from the University of Bern, Dr Alexander Wong, Dr Katie Bayfield, Anneliese Blaxland and Imogen Byrne from The Children's Hospital at Westmead, and Professor Wilfried Karmaus from the University of Memphis for comments.

Contributors JM, UF, PL, PDR and AMC conceived the project and supervised the lung function analysis. PGG, VEM and JM conducted the Breathing for Life Trial (BLT) pregnancy study. JM, PGG, VEM, AMC, PDR, PDS and KH conducted the BLT infant follow-up. PDGB, KJ and EDQA performed the infant lung function. JM supervised the BLT infant follow-up. UF and PL supervised the Bern Infant Lung Development (BILD) study follow-up. UF, PL, RA, OG, OF and JU conducted the BILD infant follow-up. CO and GMCG performed the multivariable Bayesian regression analyses. CO supervised all other statistical analyses and wrote the statistical method section. PDGB and EDQA analysed all lung function data. PDGB and JM wrote a draft manuscript. All authors edited the final version of the manuscript.

Funding The study was funded by the National Health and Medical Research Council, Hunter Children's Research Foundation; Hunter Medical Research Institute, PRC GrowUpWell University of Newcastle; and Charitable Trust, John Hunter Hospital.

Competing interests None declared.

\section{Patient consent for publication Not required.}

Ethics approval The BLT study was approved by the Hunter New England Human Research Ethics Committee of the Hunter New England Local Health District (ref no 12/10/17/3.04). The BILD study was approved by The Bernese Cantonal Ethics Research Committee (Bern, Switzerland).

Provenance and peer review Not commissioned; externally peer reviewed. Data availability statement Data are available upon reasonable request.

\section{ORCID iDs}

Adam M Collison http://orcid.org/0000-0002-3074-3046

Vanessa E Murphy http://orcid.org/0000-0003-3282-1324

Paul D Robinson http://orcid.org/0000-0001-7397-105X

Peter D Sly http://orcid.org/0000-0001-6305-2201

Jakob Usemann http://orcid.org/0000-0002-9987-2866

\section{REFERENCES}

1 Wang G, Murphy VE, Namazy J, et al. The risk of maternal and placental complications in pregnant women with asthma: a systematic review and meta-analysis. J Matern Fetal Neonatal Med 2014;27:934-42.

2 Kemppainen M, Lahesmaa-Korpinen A-M, Kauppi P, et al. Maternal asthma is associated with increased risk of perinatal mortality. PLoS One 2018;13:e0197593.

3 Vanders RL, Murphy VE. Maternal complications and the management of asthma in pregnancy. Womens Health 2015;11:183-91. 
4 Abdullah K, Zhu J, Gershon A, et al. Effect of asthma exacerbation during pregnancy in women with asthma: a population-based cohort study. Eur Respir J 2020;55:1901335.

5 Fuchs $\mathrm{O}$, Latzin $\mathrm{P}$, Thamrin C, et al. Normative data for lung function and exhaled nitric oxide in unsedated healthy infants. Eur Respir J 2011;37:1208-16.

6 Stick SM, Burton PR, Gurrin L, et al. Effects of maternal smoking during pregnancy and a family history of asthma on respiratory function in newborn infants. The Lancet 1996:348:1060-4.

7 Hall GL. Smoking during pregnancy, vitamin C supplementation, and infant respiratory health. JAMA 2014;311:2070-1.

8 Martinez FD. Role of respiratory infection in onset of asthma and chronic obstructive pulmonary disease. Clin Exp Allergy 1999;29 Suppl 2:53-8.

9 Tagiyeva N, Devereux G, Fielding S, et al. Outcomes of childhood asthma and wheezy bronchitis. A 50-year cohort study. Am J Respir Crit Care Med 2016;193:23-30.

10 McGeachie MJ, Yates KP, Zhou X, et al. Patterns of growth and decline in lung function in persistent childhood asthma. N Eng/ J Med 2016;374:1842-52.

11 Murphy VE, Jensen ME, Mattes J, et al. The Breathing for Life Trial: a randomised controlled trial of fractional exhaled nitric oxide (FENO)-based management of asthma during pregnancy and its impact on perinatal outcomes and infant and childhood respiratory health. BMC Pregnancy Childbirth 2016;16:111.

12 Bates JHT, Peters U. A model-based approach to interpreting multibreath nitrogen washout data. J Appl Physiol 2018;124:1155-63.

13 Frey U, Stocks J, Coates A, et al. Specifications for equipment used for infant pulmonary function testing. ERS/ATS Task force on standards for infant respiratory function testing. European respiratory Society/ American thoracic Society. Eur Respir J 2000;16:731-40.

14 Gray D, Willemse L, Visagie A, et al. Lung function and exhaled nitric oxide in healthy unsedated African infants. Respirology 2015;20:1108-14.

15 Bates JH, Schmalisch G, Filbrun D, et al. Tidal breath analysis for infant pulmonary function testing. ERS/ATS Task force on standards for infant respiratory function testing. European respiratory Society/American thoracic Society. Eur Respir $J$ 2000;16:1180-92.

16 Team RC. R: A language and environment for statistical computing. In: Computing RFfS. Vienna, Austria, 2020.

17 Gelman A. Prior distributions for variance parameters in hierarchical models. Bayesian Data Analysis 2006;1:515-33.

18 Jeffreys H. The theory of probability. 3rd ed. Oxford, England, 1998: 470.

19 Bürkner P-C. brms : An R Package for Bayesian Multilevel Models Using Stan. J Stat Softw 2017:80:21705

20 Gray D, Willemse L, Visagie A, et al. Determinants of early-life lung function in African infants. Thorax 2017;72:445-50
21 Bentsen MH, Markestad T, Øymar K, et al. Lung function at term in extremely pretermborn infants: a regional prospective cohort study. BMJ Open 2017;7:e016868.

22 Nielsen HC, Zinman HM, Torday JS. Dihydrotestosterone inhibits fetal rabbit pulmonary surfactant production. J. Clin. Invest. 1982;69:611-6.

23 Levesque BM, Vosatka RJ, Nielsen HC. Dihydrotestosterone stimulates branching morphogenesis, cell proliferation, and programmed cell death in mouse embryonic lung explants. Pediatr Res 2000;47:481-91.

24 Kho AT, Chhabra D, Sharma S, et al. Age, sexual dimorphism, and disease associations in the developing human fetal lung transcriptome. Am J Respir Cell Mol Biol 2016;54:814-21.

25 Belgrave DCM, Granell R, Turner SW, et al. Lung function trajectories from pre-school age to adulthood and their associations with early life factors: a retrospective analysis of three population-based birth cohort studies. Lancet Respir Med 2018;6:526-34.

26 Murray CS, Pipis SD, McArdle EC, et al. Lung function at one month of age as a risk factor for infant respiratory symptoms in a high risk population. Thorax 2002;57:388-92.

27 Drysdale SB, Wilson T, Alcazar M, et al. Lung function prior to viral lower respiratory tract infections in prematurely born infants. Thorax 2011;66:468-73.

28 Håland G, Carlsen KCL, Sandvik L, et al. Reduced lung function at birth and the risk of asthma at 10 years of age. $N$ Eng/ J Med 2006;355:1682-9.

29 Bisgaard $\mathrm{H}$, Jensen SM, Bønnelykke K. Interaction between asthma and lung function growth in early life. Am J Respir Crit Care Med 2012;185:1183-9.

30 Tse SM, Rifas-Shiman SL, Coull BA, et al. Sex-Specific risk factors for childhood wheeze and longitudinal phenotypes of wheeze. J Allergy Clin Immunol 2016:138:1561-8.

31 Juhn YJ, Weaver A, Katusic S, et al. Mode of delivery at birth and development of asthma: a population-based cohort study. J Allergy Clin Immunol 2005;116:510-6.

32 Gerlich J, Benecke N, Peters-Weist AS, et al. Pregnancy and perinatal conditions and atopic disease prevalence in childhood and adulthood. Allergy 2018;73:1064-74.

33 McLaughlin K, Jensen M, Foureur M, et al. Antenatal asthma management by midwives in Australia - Self-reported knowledge, confidence and guideline use. Women Birth 2020;33:166-75.

34 Lebold KM, Drake MG, Hales-Beck LB, et al. IL-5 Exposure In Utero Increases Lung Nerve Density and Airway Reactivity in Adult Offspring. Am J Respir Cell Mol Biol 2020;62:493-502.

35 Drake MG, Scott GD, Blum ED, et al. Eosinophils increase airway sensory nerve density in mice and in human asthma. Sci Trans/ Med 2018;10:eaar8477.

36 Mattes J, Collison A. Fetal eosinophils get on the nerves of airways. early origins of bronchoconstriction. Am J Respir Cell Mol Biol 2020;62:407-8. 